\title{
New derivation of a third post-Newtonian equation of motion for relativistic compact binaries without ambiguity
}

\author{
Yousuke Itoh* \\ Max-Planck-Institut für Gravitationsphysik, Albert-Einstein-Institut, Am Mühlenberg 1, Golm 14476, Germany
}

Toshifumi Futamase ${ }^{\dagger}$

Astronomical Institute, Graduate School of Science, Tohoku University, Sendai, 980-8578, Japan

(Received 4 July 2003; published 1 December 2003)

\begin{abstract}
A third post-Newtonian (3PN) equation of motion for an inspiraling binary consisting of two spherical compact stars with strong internal gravity is derived under the harmonic coordinate condition using the strong field point particle limit. The equation of motion is complete in the sense that it is Lorentz invariant in the post-Newtonian perturbative sense, admits the conserved energy of the orbital motion, and is unambiguous, that is, with no undetermined coefficient. In this paper, we show explicit expressions of the 3PN equation of motion and an energy of the binary orbital motion in the case of a circular orbit (neglecting the 2.5PN radiation reaction effect) and in the center of mass frame. It is argued that the 3PN equation of motion we obtained is physically unambiguous. Full details will be reported elsewhere.
\end{abstract}

DOI: 10.1103/PhysRevD.68.121501

PACS number(s): 04.25.Nx, 04.25.-g

Renewed attention has been paid to a high order postNewtonian equation of motion governing inspiraling compact binaries in the context of the efforts for direct detection of gravitational waves $[1,2]$. It is well known that the detectability of the gravitational waves emitted by the binaries and the quality of the measurements of astrophysical information (e.g., masses) depend on the accuracy of the theoretical knowledge of the waveforms [1], and hence partly of the dynamics of the binaries.

The third post-Newtonian (3PN) approximation has been a subject of much discussion because of its ambiguity reported originally by Jaranowski and Schäfer [3]. In fact, the 3PN Arnowitt-Deser-Misner (ADM) Hamiltonian in the ADM-type gauge obtained in [3] has two undetermined coefficients $\left(\omega_{\text {kinetic }}\right.$ and $\left.\omega_{\text {static }}\right)$ and the 3PN equation of motion in the harmonic gauge derived by Blanchet and Faye [4] has one coefficient $\lambda$ undetermined within their framework. Both groups have used Dirac delta distributions, which cause divergences in general relativity, to express the point particles and inevitably they have resorted to mathematical regularizations. Damour et al. [5] pointed out that the undetermined coefficients may arise due to the unsatisfactory features of the regularizations they have used in $[3,4]$. Indeed, using dimensional regularization, Ref. [5] has succeeded in determining both of the coefficients, namely, $\omega_{\text {static }}=0$, which means $\lambda=-1987 / 3080$ via a relationship established in [6]. ( $\omega_{\text {kinetic }}$ is related to the Lorentz invariance and was fixed in $[5,7]$. Blanchet and Faye have developed a Lorentz invariant Hadamard patie finie regularization $[8,9]$ and do not have any ambiguities other than $\lambda$.)

In gravitational wave data analysis, the reduction of predictability of the equation of motion due to the undetermined coefficient can become a problem. In fact, the 3.5PN phase

\footnotetext{
*Email address: yousuke@aei.mpg.de

${ }^{\dagger}$ Email address: tof@astr.tohoku.ac.jp
}

evolution equation and luminosity [10] unfortunately have four undetermined coefficients, one of which is $\lambda$.

Theoretically, the use of Dirac delta distributions and inevitable regularization should be verified in some manner. The perfect (physical) agreements among the results obtained by various authors with various methods [11-13] give a direct theoretical confirmation of the $2.5 \mathrm{PN}$ result first derived by Damour and Deruelle [14]. It is important to achieve 3PN iteration without introducing singular sources to derive an unambiguous result and support the previous 3PN works which have used Dirac delta distributions.

Based on our previous papers $[12,15]$, we derive a $3 \mathrm{PN}$ equation of motion for two spherical compact stars in the harmonic gauge without introducing singular sources. Instead, we apply the strong field point particle limit [16] to deal with strong internal gravity of the stars. Our derivation is satisfactory in a sense that the equation admits conserved energy, is Lorentz invariant, and is unambiguous. In this paper we shall show both the 3PN equation of motion and an associated 3PN energy of the orbital motion in the center of the mass frame and in the case of a circular orbit.

Below, we shall explain briefly yet another derivation of a 3PN equation of motion. Since this method is different from others, we mention some details specific to our method at the 3PN order. After deriving an invariant energy of the binary orbital motion, we shall compare it with that derived by Blanchet and Faye and fix the $\lambda$ parameter. A full explanation of our method, including computational details, will be reported in [17]. See also [12,15].

We write explicitly the post-Newtonian expansion parameter, $\epsilon$, which represents the smallness of the orbital velocities of the stars. The mass scales as $\epsilon^{2}$ from the postNewtonian scaling. Then the strong field point particle limit [16] is achieved by setting the radius of the star to scale at the same rate as its mass, $\epsilon^{2}$. The scalings of the mass and the radius enable us to incorporate in the post-Newtonian limit $(\epsilon \rightarrow 0)$ the limit of a regular point particle whose internal gravity, approximately the mass over the radius, is strong irrelevantly to $\epsilon$. 
We derive an equation of motion via surface integrals of the gravitational energy momentum flux going through a sphere surrounding the star. For this method, we prepare two spheres $B_{A}(\tau) \equiv\left\{x^{k}|| \hat{x}-\vec{z}_{A}(\tau) \mid \leqslant \epsilon R_{A}\right\} \quad$ (A labels the two stars) on the $\tau=$ const surface, where $\tau$ is the time coordinate in the near zone. $B_{A}$, called the body zone, is centered at the star $A$ 's representative point $z_{A}^{i}(\tau)$ and has a radius $\epsilon R_{A}$, where $R_{A}$ is an arbitrary constant and is smaller than the orbital separation but larger than the radii of the stars. We make the body zone radius shrink proportionally to $\epsilon$ in the near zone coordinate $\left(\tau, x^{i}\right)$ to ensure that the field on the body zone boundary due to the star is obtained by multipole expansion when the $\epsilon$ zero limit is taken.

The $l$ th multipole moments of the star $A$ denoted by $I_{A}^{L}(\tau)$, including its mass, are defined as volume integrals over $B_{A}$ of $\Lambda^{\tau \tau} \equiv-g\left(T^{\tau \tau}+t_{L L}^{\tau \tau}\right)+\chi^{\tau \tau \alpha \beta}{ }_{, \alpha \beta}$ where $g, T^{\mu \nu}$ and $t_{L L}^{\mu \nu}$ are the determinants of the metric $g_{\mu \nu}$, the matter stress energy tensor and the Landau-Lifshitz pseudo tensor. $\chi^{\mu \nu \alpha \beta}{ }_{, \alpha \beta}$ arises since we use the wave operator of the flat spacetime instead of that of the curved spacetime when we solve the harmonically relaxed Einstein equation,

$$
I_{A}^{L} \equiv \epsilon^{2} \int_{B_{A}(\tau)} d^{3} \alpha_{A} \Lambda^{\tau \tau} \alpha_{A}^{L},
$$

where we introduced the body zone coordinate $\alpha_{A}^{i}=\epsilon^{-2}\left[x^{i}\right.$ $\left.-z_{A}^{i}(\tau)\right]$ and multi-indices notation $L=i_{1} \cdots i_{l}$ ( $l \geqslant 0$ : integer). $\alpha_{A}^{L}=\alpha_{A}^{i_{1}} \cdots \alpha_{A}^{i_{l}}$. The mass $m_{A} \equiv \lim _{\epsilon \rightarrow 0} P_{A}^{\tau}$ with $P_{A}^{\tau}$ $\equiv I_{A}^{0}$ so defined would be the ADM mass if the companion star were absent and the body zone radius is taken to be infinite in the body zone coordinate.

In deriving a 3PN equation of motion, it is important in our formalism to notice that the body zone $B_{A}$ is a sphere in the frame where the star $A$ orbits, but $B_{A}$ is not a sphere in the generalized Fermi frame [20] where the star $A$ is at rest and the effect of the gravitational field due to the companion star is removed (as much as possible) except for, namely, the tidal effect. We define stars to be spherical in the generalized Fermi frame. Now, we define the "intrinsic" multipole moments $\hat{I}_{A}^{L}(\hat{\tau})$ on the $\hat{\tau}=$ const surface in the generalized Fermi coordinate $\left(\hat{\tau}, \hat{x}^{i}\right)$ as a volume integral over a sphere $\hat{B}_{A}(\hat{\tau})$ centered at the same world event and with the same radius $\epsilon R_{A}$ as $B_{A}(\tau)$. For example, the difference between the symmetric tracefree quadrupole moments is defined as $\epsilon^{8} \delta I_{A}^{\langle i j\rangle} \equiv \epsilon^{8} I_{A}^{\langle i j\rangle}-\epsilon^{8} \hat{I}_{A}^{\langle i j\rangle}=\int_{B_{A}(\tau)} d^{3} y_{A} \Lambda^{\tau \tau} y_{A}^{\langle i} y^{j\rangle}$

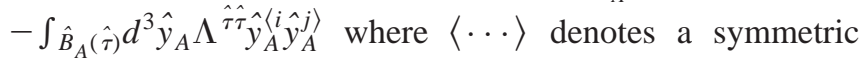
tracefree operation on the indices between the brackets. For "spherically symmetric" compact stars, the "intrinsic" tracefree quadrupole moment $\hat{I}_{A}^{\langle i j\rangle}$ vanishes. Up to the 3PN iteration of the gravitational field, $\delta I_{A}^{\langle i j\rangle}$ arise mainly due to the Lorentz contraction and can be evaluated as a surface integral over $\partial B_{A}$,

$$
\begin{aligned}
\delta I_{A}^{\langle i j\rangle} & =\epsilon^{-6} \frac{1}{2} v_{A}^{k} v_{A}^{l} \oint_{\partial B_{A}} d S_{k} y_{A}^{l} y_{A}^{\langle i} y_{A}^{j\rangle} \Lambda^{\tau \tau}+O\left(\epsilon^{4}\right) \\
& =-\epsilon^{2} \frac{4}{5} m_{A}^{3} v_{A}^{\langle i} v_{A}^{j\rangle}+O\left(\epsilon^{4}\right) .
\end{aligned}
$$

In our formalism, it is possible to derive the 3PN field for an isolated star (by taking a limit where the mass of the companion star is zero). $\delta I_{A}^{\langle i j\rangle}$ is a necessary term to obtain the correct expression of the 3PN field for such a system. Although other multipole moments defined over $B_{A}$ possibly hide purely monopole terms, only the quadrupole moment is found to be relevant up to the 3PN order. Clean extraction of monopole terms from the multipole moments defined in our previous works is a problem at the 3PN order specific to our formalism. Blanchet and Faye, on the other hand, elaborate their generalized Hadamard partie finie regularization [8] in a Lorentz invariant manner [9], and have properly taken into account special relativistic kinematic effects, including the Lorentz contraction.

Now, let us briefly explain our derivation of the equation of motion. The local conservation law of the total energy gives an evolution equation for four-momentum of the star and relationships among the multipole moments, namely, momentum-velocity relation. The last reads as $P_{A}^{i}=P_{A}^{\tau} v_{A}^{i}$ $+Q_{A}^{i}+\epsilon^{2} d D_{A}^{i} / d \tau$ where $P_{A}^{i} \equiv \epsilon^{2} \int_{B_{A}} d^{3} \alpha_{A} \Lambda^{\tau i}$ and $P_{A}^{\tau}$ are the three-momentum and the energy of the star $A . Q_{A}^{i}$ $\equiv \epsilon^{-4} \oint_{B_{A}} d S_{k}\left(\Lambda^{\tau k}-v_{A}^{k} \Lambda^{\tau \tau}\right) y_{A}^{i}$ arises since the (pseudo-) stress energy momentum of the field extends outside of the star [12]. $Q_{A}^{i}$ can be evaluated explicitly and do contribute to the 3 PN velocity momentum relation. We can define the representative point $z_{A}^{i}(\tau)$ of the star $A$ by specifying the dipole moment of the star, $D_{A}^{i} \equiv I_{A}^{i}$ [e.g., $z_{A}^{i}(\tau)$ corresponding to a condition $D_{A}^{i}=0$ may be called the center of mass of the star $A$ from an analogy of the Newtonian dynamics]. The relationship between the energy $P_{A}^{\tau}$ and the mass $m_{A}$ can be obtained by integrating functionally the evolution equation of $P_{A}^{\tau}$, which is expressed as surface integrals and can be evaluated explicitly up to the $3 \mathrm{PN}$ order, in the form as $P_{A}^{\tau}$ $=m_{A}\left[1+O\left(\epsilon^{2}\right)\right]$.

Combining the mass energy relation, the momentum velocity relation, and the evolution equation for the fourmomentum, we obtain the general form of the equation of motion [12],

$$
\begin{aligned}
m_{A} \frac{d v_{A}^{i}}{d \tau}= & -\epsilon^{-4} \oint_{\partial B_{A}} d S_{k} \Lambda^{k i}+\epsilon^{-4} v_{A}^{k} \oint_{\partial B_{A}} d S_{k} \Lambda^{\tau i} \\
& +\epsilon^{-4} v_{A}^{i}\left(\oint_{\partial B_{A}} d S_{k} \Lambda^{k \tau}-v_{A}^{k} \oint_{\partial B_{A}} d S_{k} \Lambda^{\tau \tau}\right) \\
& -\frac{d Q_{A}^{i}}{d \tau}-\epsilon^{2} \frac{d^{2} D_{A}^{i}}{d \tau^{2}}+\left(m_{A}-P_{A}^{\tau}\right) \frac{d v_{A}^{i}}{d \tau}
\end{aligned}
$$

Note that $\Lambda^{\mu \nu}=(-g) t_{L L}^{\mu \nu}+\chi^{\mu \nu \alpha \beta}{ }_{\alpha \beta}$ on $\partial B_{A}$, since $\partial B_{A}$ is well outside the star by construction of the body zone. The acceleration in the right hand side of Eq. (3) should be un- 
derstood to be lower order acceleration than in the left hand side. The terms in the right hand side of Eq. (3) are completely expressed as surface integrals over the body zone boundary except for $D_{A}^{i}$ to be specified. The surface integral approach enables us to derive an equation of motion irrelevant to the internal structure of the star. (Effects of the star's internal structure on the orbital motion such as tidally induced multipole moments appear through the field and hence the integrand $\Lambda^{\mu \nu}$.) The scaling of the body zone radius $\epsilon R_{A}$ ensures that we have an equation of motion for compact stars.

The field equation coupled to the matter equations mentioned above is the integrated relaxed Einstein equation under harmonic gauge $\left[h^{\mu \nu} \equiv \eta^{\mu \nu}-\sqrt{-g} g^{\mu \nu}\right.$ where $\eta^{\mu \nu}$ $=\operatorname{diag}\left(-\epsilon^{2}, 1,1,1\right)$. The harmonic condition is then $h^{\mu \nu}{ }_{, \nu}$ $=0$ ]

$$
h^{\mu \nu}\left(\tau, x^{i}\right)=4 \int_{C\left(\tau, x^{i}\right)} d^{3} y \frac{\Lambda^{\mu \nu}\left(\tau-\epsilon|\vec{x}-\vec{y}|, y^{k} ; \epsilon\right)}{|\vec{x}-\vec{y}|} .
$$

We split the flat light cone $C\left(\tau, x^{i}\right)$ into four parts: two body zones $B_{A}$, a near zone outside the body zones $N / B$ surrounding the binary and the far zone outside the near zone. For $B_{A}$ and $N / B$ contributions to the field $h^{\mu \nu}\left(\tau, x^{i}\right)$, we expand the retarded field about the near zone time $\tau$. Then multipole expansion of the star is used to evaluate the two body zone contributions. The $N / B$ contribution is basically evaluated with the help of superpotentials (a superpotential here means a particular solution valid in $N / B$ of a Poisson equation with a noncompact support source in $N / B$ ). Unfortunately, it was not possible to find all the necessary superpotentials explicitly at the 3PN order. For integrands in Eq. (4) such that we could not find superpotentials in closed forms, after making the retarded expansion we leave the Poisson integrals unevaluated and substitute the (not-integrated) field into $\Lambda^{\mu \nu}$ in Eq. (3). Then we perform the surface integrals in Eq. (3) [with respect to the spatial variable $x^{i}$ in Eq. (4)] first and next perform the remaining volume integrals [with respect to the spatial variable $y^{i}$ in Eq. (4)]. In other words, we extract the parts of the field necessary to derive the equation of motion by interchanging the order of the integrations in Eq. (3) and Eq. (4). As a check, we applied this method on the integrands for which the necessary superpotentials can be derived in closed forms, and found that both methods give the same result. Finally, we have dealt with the integration over the far zone using the direct integration of the relaxed Einstein equations method $[13,18]$ and found that it does not contribute to the $3 \mathrm{PN}$ equation of motion $[13,19]$.

Using the method mentioned above, we obtain a 3PN equation of motion for a two-spherical compact stars binary. We here present the 3PN relative acceleration in the case of the circular orbit and in the center of mass frame, which is an appropriate equation to inspiraling binaries,

$$
\frac{d V^{i}}{d \tau}=-\Omega_{\ln }^{2} r_{12}^{i}+\epsilon_{2.5 \mathrm{PN}}^{5} A^{i},
$$

where $V^{i}=v_{1}^{i}-v_{2}^{i}$ is the relative velocity and $2.5 \mathrm{PN}^{i}$ is the relative acceleration at the $2.5 \mathrm{PN}$ order (the radiation reaction term). The 3PN orbital angular frequency $\Omega_{\ln }$ is (for comparison, we adopt similar notations as in [4])

$$
\begin{aligned}
m^{2} \Omega_{\ln }^{2}= & \gamma^{3}\left[1+\epsilon^{2} \gamma(-3+\nu)+\epsilon^{4} \gamma^{2}\left(6+\frac{41}{4} \nu+\nu^{2}\right)\right. \\
& +\epsilon^{6} \gamma^{3}\left(-10+\nu\left\{-\frac{2375}{24}+\frac{41 \pi^{2}}{64}+22 \ln \left(\frac{r_{12}}{R_{0}}\right)\right\}\right. \\
& \left.\left.+\frac{19}{2} \nu^{2}+\nu^{3}\right)\right]+O\left(\epsilon^{7}\right),
\end{aligned}
$$

where $m=m_{1}+m_{2}, \quad \nu=m_{1} m_{2} / m^{2}, \quad \gamma=m / r_{12}$, and $\ln R_{0}$ $=\left[m_{1} \ln \left(\epsilon R_{1}\right)+m_{2} \ln \left(\epsilon R_{2}\right)\right] / m$. In Eq. (5) with (6), the representative point of the star $A, z_{A}^{i}(\tau)$, is defined by setting $D_{A}^{i}=\epsilon^{4} \delta_{A Q}^{i}=-86 \epsilon^{4} m_{A N}^{3} a_{A}^{i} / 9$, where ${ }_{N} a_{A}^{i}$ is the Newtonian acceleration. This choice makes the three-momentum $P_{A}^{i}$ parallel to the velocity $v_{A}^{i}$. We note that there is no arbitrary parameter other than the body zone radii $\epsilon R_{A}$ in the 3PN relative acceleration. We here note that it is not allowed to fix the $\lambda$ parameter by comparing Eq. (6) with the corresponding result of Blanchet and Faye [4], since the harmonic condition both groups have used does not fix the gauge completely [17] and the expression of the 3PN orbital angular frequency in terms of the coordinate distance $\mathrm{m} / \mathrm{r}_{12}$, Eq. (6), is gauge dependent. [For the same reason, we cannot fix $\lambda$ using Eq. (13).]

We can remove away $\epsilon R_{A}$ dependence from the 3PN relative acceleration, Eq. (5), physically by a suitable redefinition of the representative points of the stars. In fact, by setting

$$
D_{A, \mathrm{New}}^{i}=\epsilon^{4} \delta_{A Q}^{i}-\epsilon^{4} \frac{22}{3} m_{A N}^{3} a_{A}^{i} \ln \left(\frac{r_{12}}{\epsilon R_{A}}\right)
$$

we obtain the 3PN relative acceleration free from any arbitrary parameter

$$
\frac{d V^{i}}{d \tau}=-\Omega^{2} r_{12}^{i}+\epsilon_{2.5 \mathrm{PN}}^{5} A^{i},
$$

with $m^{2} \Omega^{2}=m^{2} \Omega_{\ln }^{2}-22 \epsilon^{6} \gamma^{6} \nu \ln \left(r_{12} / R_{0}\right)$. This observation, in fact, is the case in general cases (i.e., in general orbits not in the center of mass frame); the 3PN equation of motion in general cases we have derived is physically free from any ambiguity.

A reason why we are concerned with $\ln \epsilon R_{A}$ dependence is the following. Blanchet and Faye have introduced four arbitrary parameters in their regularization procedure, two of which appear in the regularization of the field having two singular points, and the others appear in the regularization of equations of motion for those two points. They showed that the two of those parameters can be gauged away, while the other two were consumed to make their equations of motion conservative (modulo the radiation reaction effect), and they found there remained one and only one parameter $\lambda$, although the relationship between energy conservation and 
regularization parameters associated with a point particle description is not clear. Our redefinition of the representative points (7) corresponds to their gauge transformation. Then, their observation makes us check if it is physically allowed to remove the $\ln \epsilon R_{A}$ dependence in our 3PN equation of motion, since we introduced only two arbitrary parameters $\epsilon R_{A}$ and we have no freedom to make our equation motion conservative by adjusting these two parameters if we remove them away. Thus, we have two problems to be solved in our method: removal of $\ln \epsilon R_{A}$ and an energy conservation. For lack of space, here we show some facts which support naturality of Eq. (7). The energy conservation problem will be addressed in [17]; there we shall show our equation of motion and an associated conserved energy of the binary orbital motion in general cases.

Let us consider the harmonic condition

$$
\begin{aligned}
0= & h_{, \mu}^{\tau \mu}=4 \epsilon^{4} \sum_{A=1,2}\left[\frac{1}{r_{A}} \frac{d P_{A}^{\tau}}{d \tau}+\frac{r_{A}^{i}}{r_{A}^{3}}\left(P_{A}^{\tau} v_{A}^{i}+\epsilon^{2} \frac{d D_{A}^{i}}{d \tau}-P_{A}^{i}\right)\right. \\
& \left.+\sum_{A=1,2} \oint_{\partial B_{A}} \frac{d S_{i}}{|\vec{x}-\vec{y}|}\left(\Lambda^{\tau i}-v_{A}^{i} \Lambda^{\tau \tau}\right)+\cdots\right] \\
0= & h^{i \mu}, \mu \\
= & {\left[\epsilon^{4} \sum_{A=1,2} \frac{1}{r_{A}} \frac{d P_{A}^{i}}{d \tau}+\sum_{A=1,2} \oint_{\partial B_{A}} \frac{d S_{j}}{|\vec{x}-\vec{y}|}\right.} \\
& \left.\times\left(\Lambda^{i j}-v_{A}^{j} \Lambda^{\tau i}\right)+\cdots\right]
\end{aligned}
$$

where "..." are irrelevant terms. These equations are a manifestation of the fact that the harmonic condition is consistent with the evolution equation of $P_{A}^{\tau}$, the momentumvelocity relation, and the equation of motion (and relations among higher multipole moments, hidden in "..."). Thus, if logarithmic dependence of $\epsilon R_{A}$ arises from the equation of motion [essentially the second term of Eq. (10)], $P_{A}^{i}$ must have the same logarithmic dependence (times minus sign) to ensure harmonicity. This and the momentum velocity relation in turn mean $P_{A}^{\tau}, v_{A}^{i}=d z_{A}^{i} / d \tau$ or $D_{A}^{i}$ have corresponding logarithmic dependence. We found [17] that $P_{A}^{\tau}$ have no logarithm up to the 3PN order. Therefore $z_{A}^{i}$ or $D_{A}^{i}$ should have logarithms. This is consistent with the fact that a choice of $D_{A}^{i}$ determines $z_{A}^{i}$. $z_{A}^{i}$ depends on logarithms if the old choice is taken, while it does not if our new choice is taken.

The second fact that supports our interpretation is as follows. We find that the near zone dipole moment $D_{N}^{i}$ defined by a volume integral of $\Lambda^{\tau \tau} y^{i}$ becomes

$$
\begin{aligned}
\epsilon^{2} D_{N}^{i} \equiv & \epsilon^{-4} \int_{N} d^{3} y \Lambda^{\tau \tau} y^{i}=\sum_{A=1,2} P_{A}^{\tau} z_{A}^{i}+\epsilon^{2} \sum_{A=1,2} D_{A}^{i} \\
& +\epsilon^{-4} \int_{N / B} d^{3} y \Lambda^{\tau \tau} y^{i}
\end{aligned}
$$

Then if we take the old choice of $D_{A}^{i}$, the volume integral becomes

$$
\int_{N / B} d^{3} y \Lambda^{\tau \tau} y^{i}=\epsilon^{4} \frac{22}{3} \sum_{A=1,2} m_{A N}^{3} a_{A}^{i} \ln \left(\frac{r_{12}}{\epsilon R_{A}}\right)+\cdots
$$

where terms denoted by "..." are independent of $R_{A}$. Notice that the near zone dipole moment can be freely determined, say, $D_{N}^{i}=0$, since we can define the origin of the near zone freely in general [21]. By taking temporal derivatives of $D_{N}^{i}$ twice, we see that $D_{A \text { New }}^{i}$ gives the definition of $z_{A}^{i}(\tau)$ in terms of which the 3PN equation of motion is independent of $\epsilon R_{A}$.

Finally, we show the 3PN conserved energy (neglecting the 2.5PN radiation reaction force) of the circular orbital motion in the center of mass frame. Using Eq. (5), we have

$$
\begin{aligned}
E_{\ln }(\gamma)= & -\frac{m \nu \gamma}{2}\left(1+\epsilon^{2} \gamma\left(-\frac{7}{4}+\frac{\nu}{4}\right)\right. \\
& +\epsilon^{4} \gamma^{2}\left(-\frac{7}{8}+\frac{49}{8} \nu+\frac{1}{8} \nu^{2}\right) \\
& +\epsilon^{6} \gamma^{3}\left[-\frac{235}{64}+\frac{27}{32} \nu^{2}+\frac{5}{64} \nu^{3}\right. \\
& \left.\left.+\nu\left\{\frac{10141}{576}-\frac{123 \pi^{2}}{64}+\frac{22}{3} \ln \left(\frac{r_{12}}{R_{0}}\right)\right\}\right]\right)+O\left(\epsilon^{7}\right) .
\end{aligned}
$$

In terms of $x=\left(m \Omega_{\mathrm{ln}}\right)^{2 / 3}$ we obtain the 3PN energy in an invariant form

$$
\begin{aligned}
E_{\ln }(x)= & -\frac{m \nu x}{2}\left[1+\epsilon^{2}\left(-\frac{3}{4}-\frac{1}{12} \nu\right) x\right. \\
& +\epsilon^{4}\left(-\frac{27}{8}+\frac{19}{8} \nu-\frac{1}{24} \nu^{2}\right) x^{2} \\
& +\epsilon^{6}\left(-\frac{675}{64}+\left\{\frac{34445}{576}-\frac{205 \pi^{2}}{96}\right\} \nu\right. \\
& \left.\left.-\frac{155}{96} \nu^{2}-\frac{35}{5184} \nu^{3}\right) x^{3}\right]+O\left(\epsilon^{7}\right) .
\end{aligned}
$$

Similarly, using Eq. (8), we have $E(\gamma)=E_{\ln }(\gamma)$ $+(11 / 3) \epsilon^{6} m \nu^{2} \gamma^{4} \ln \left(r_{12} / R_{0}\right)$. Here we note that the difference between $E(\gamma)$ and $E_{\ln }(\gamma)$ is merely due to the redefinition of the dipole moments (or equivalently, a coordinate transformation under the harmonic coordinate condition). The invariant energy $E(x)$ is the same as $E_{\ln }(x)$ but with $x$ $=\left(m \Omega_{\ln }\right)^{2 / 3}$ replaced with $x=(m \Omega)^{2 / 3}$. This third fact, that the energy has the same form for both definitions of the representative points of the stars when we write the energy in terms of the orbital angular frequency, which is an observable, supports that the apparent body zone radii dependence of the 3PN relative acceleration has no physical effect on the orbital motion.

We have thus derived a 3PN equation of motion which takes account of strong internal gravity of the stars and 
avoids any ambiguity. Comparing our result, Eq. (14), with the corresponding result in [4], we determine the coefficient undetermined in the Blanchet and Faye 3PN equation of motion as $\lambda=-1987 / 3080$. This value of $\lambda$ is consistent with the result of Damour, Jaranowski, and Schäfer [5]. Thus, our result (indirectly) validates their use of the dimensional regularization in the ADM Hamiltonian approach in the ADM transverse traceless gauge. Finally, we note that
Blanchet et al. [22] have recently obtained the same value of $\lambda$, and computed a 3PN equation of motion in the harmonic gauge using dimensional regularization.

Y.I. was partly supported by the JSPS. The authors would like to acknowledge $\mathrm{H}$. Asada for fruitful discussion and comments. Extensive use has been made of the software MATHEMATICA and MATHTENSOR.
[1] C. Cutler et al., Phys. Rev. Lett. 70, 2984 (1993); E. Poisson, Phys. Rev. D 55, 7980 (1997); T. Tanaka, H. Tagoshi, and M. Sasaki, Prog. Theor. Phys. 96, 1087 (1996).

[2] L. Blanchet, Living Rev. Relativ. 5, 3 (2002).

[3] P. Jaranowski and G. Schäfer, Phys. Rev. D 57, 7274 (1998); 63, 029902(E) (2001); 57, 5948 (1998); 60, 124003 (1999).

[4] L. Blanchet and G. Faye, Phys. Lett. A 271, 58 (2000); Phys. Rev. D 63, 062005 (2001).

[5] T. Damour, P. Jaranowski, and G. Schäfer, Phys. Lett. B 513, 147 (2001).

[6] T. Damour, P. Jaranowski, and G. Schäfer, Phys. Rev. D 63, 044021 (2001); 66, 029901(E) (2002); V.C. de Andrade, L. Blanchet, and G. Faye, Class. Quantum Grav. 18, 753 (2001).

[7] T. Damour, P. Jaranowski, and G. Schäfer, Phys. Rev. D 62, 021501(R) (2000); 63, 029903(E) (2001).

[8] L. Blanchet and G. Faye, J. Math. Phys. 41, 7675 (2000).

[9] L. Blanchet and G. Faye, J. Math. Phys. 42, 4391 (2001).

[10] L. Blanchet, G. Faye, B.R. Iyer, and B. Joguet, Phys. Rev. D 65, 061501(R) (2002); L. Blanchet, B.R. Iyer, and B. Joguet, ibid. 65, 064005 (2002).

[11] L.P. Grishchuk and S.M. Kopejkin, Sov. Astron. Lett. 9, 230 (1983); S.M. Kopejkin, Sov. Astron. 29, 516 (1985); G. Schä- fer, Appl. Phys. (N.Y.) 161, 81 (1985); Gen. Relativ. Gravit. 18, 255 (1986); L. Blanchet, G. Faye, and B. Ponsot, Phys. Rev. D 58, 124002 (1998).

[12] Y. Itoh, T. Futamase, and H. Asada, Phys. Rev. D 63, 064038 (2001).

[13] M.E. Pati and C.M. Will, Phys. Rev. D 65, 104008 (2002).

[14] T. Damour and N. Deruelle, Phys. Lett. 87A, 81 (1981); T. Damour, C. R. Seances Acad. Sci., Ser. 2 294, 1355 (1982).

[15] Y. Itoh, T. Futamase, and H. Asada, Phys. Rev. D 62, 064002 (2000).

[16] T. Futamase, Phys. Rev. D 36, 321 (1987).

[17] Y. Itoh, "Equation of motion for relativistic compact binaries with the strong field point particle limit: Third post-Newtonian order," gr-qc/0310029.

[18] C.M. Will and A.G. Wiseman, Phys. Rev. D 54, 4813 (1996); M.E. Pati and C.M. Will, ibid. 62, 124015 (2000).

[19] L. Blanchet and T. Damour, Phys. Rev. D 37, 1410 (1988).

[20] N. Ashby and B. Bertotti, Phys. Rev. D 34, 2246 (1986).

[21] We here do not discuss an appropriate definition of the center of mass frame of the binary system.

[22] L. Blanchet (private communication). 